\title{
Implementing the teen marijuana check-up in schools-a study protocol
}

\author{
Bryan Hartzler ${ }^{1 *}$ D, Aaron R. Lyon², Denise D. Walker ${ }^{3}$, Lauren Matthews ${ }^{3}$, Kevin M. King ${ }^{4}$ \\ and Kathryn E. McCollister ${ }^{5}$
}

\begin{abstract}
Background: Substance misuse is now encountered in settings beyond addiction specialty care, with schools a point-of-contact for student access to behavioral health services. Marijuana is a leading impetus for adolescent treatment admissions despite declining risk perception, for which the Teen Marijuana Check-Up (TMCU)—a tailored adaptation of motivational enhancement therapy-offers an efficacious service option. To bridge the knowledge gap concerning effective and affordable technical assistance strategies for implementing empirically supported services, the described trial will test such a strategy to facilitate school-based TMCU implementation.
\end{abstract}

Methods: A type II effectiveness/implementation hybrid trial will test a novel strategy for a TMCU purveyor to provide technical assistance on an 'as-needed' basis when triggered by a fidelity drift alarm bell, compared to resource-intensive 'gold-standard' technical assistance procedures of prior efficacy trials. Trial procedures adhere to the EPIS framework as follows: (1) initial mixed-method exploration of the involved school contexts and identification of TMCU interventionist candidates in elicitation interviews; (2) interventionist preparation via a formally evaluated training process involving a two-day workshop and sequence of three training cases; (3) post-training implementation for 24 months for which trained interventionists are randomized to 'as-needed' or 'gold-standard' technical assistance and self-referring students randomized (in 2:1 ratio) to TMCU or waitlist/control; and (4) examination of TMCU sustainment via interventionist completion of biannual outcome assessments, cost analyses, and exit interviews. Hypothesized effects include non-differential influence of the competing technical assistance methods on both TMCU fidelity and intervention effectiveness, with lesser school costs for the 'as-needed' than 'gold-standard' technical assistance and greater reduction in the frequency of marijuana use expected among TMCU-exposed students relative to those assigned to waitlist/control.

Discussion: This trial-occurring in Washington state as legislative, fiscal, and sociocultural forces converge to heighten exposure of American adolescents to marijuana-related harms - is set to advance understanding of best implementation practices for this and other efficacious, school-based interventions through examination of a data-driven technical assistance method. If shown to be clinically useful and affordable, the concept of a fidelity drift alarm could be readily translated to other empirically supported services and in other health settings.

Trial registration: ClinicalTrials.gov NCT03111667 registered 7 April 2017.

Keywords: Technical assistance, Therapy training, EBP implementation, Fidelity, Motivational enhancement therapy, Adolescent marijuana use

\footnotetext{
* Correspondence: hartzb@u.washington.edu

${ }^{1}$ Alcohol and Drug Abuse Institute, University of Washington, 1107 NE 45th

Street, Suite 120, Seattle, WA 98105, USA

Full list of author information is available at the end of the article
} 


\section{Background}

Traditional health service research processes contribute to a 17-year lag between empirical validation and community implementation [1-3]. A common caveat of trials that establish efficacy of a given health service is the resource-intensive manner in which selected research therapists are trained and supervised to ensure the health service is delivered with fidelity so its hypothesized effects are adequately demonstrated [4]. Replication of these training and technical assistance procedures to support community-based implementation is unrealistic in most settings. Consequent need for effective, affordable strategies to support community implementation of empirically supported health services casts a shadow over the prospect of their transport to community settings, including those wherein substance misuse is encountered. Notably, legislative policies in the United States of America (i.e., Mental Health Parity and Addiction Equity Act, 2008; Patient Protection and Affordable Care Act, 2010) have diversified such settings [5].

\section{'Science-to-practice gaps' in the addiction field extend to adolescent treatments and settings}

Dating back two decades to a widely cited Institute of Medicine report [6], a persisting focus in the addiction field is bridging 'science-to-practice gaps.' As estimated by the 2014 National Survey on Drug Use and Health, a majority of the 1.3 million American adolescents with a substance use disorder struggle with marijuana specifically [7]. Further, marijuana is a leading impetus for treatment admissions [8], and linked to lesser neuropsychological functioning and academic performance [9] and greater likelihood of school dropout, emergent psychopathology, and suicidality $[10,11]$. Nevertheless, risk perceptions about marijuana use have declined among high school students [12], perhaps a function of increasingly progressive legislation widening its public availability and permissibility [13]. Consequent demand for cost-effective, clinically useful marijuana-focused treatments for adolescents is high.

Historically, addiction research has focused on adults, as evident in prominent trials comparing empirically supported therapies [14] or evaluating their combination with medications [15]. Also of primary focus has been the addiction specialty care context, as evidenced in three dozen multisite protocols conducted by the National Institute on Drug Abuse via its Clinical Trials Network [16]. Expanded inclusion of substance use disorders among essential conditions for which Americans may access health services has prompted treatment-seeking in other settings that serve as a point-of-contact [17]. Schools are an example as $70-80 \%$ of adolescents who access services doing so in schools [18]. Most school-based services lack empirical support $[19,20]$, and introduction of empirically supported services (if effectively implemented by school personnel) may substantively improve public health efforts [21, 22].

\section{Conceptual and empirical basis for the teen marijuana check-up}

Among prominent evidence-based therapies is Motivational Interviewing (MI), a "collaborative conversation style for strengthening a person's own motivation and commitment to change" [23]. An oft-studied brief adaptation of MI is Motivational Enhancement Therapy (MET), typically structured as 1-2 individual sessions wherein personal assessment data is gathered and explored [24]. This brief structure and hallmark client-centered care philosophy were core features of a popular alcoholfocused approach, the Drinker's Check-Up [25], which spurred similar self-referral interventions for specific populations and behaviors [26]. Among these is a schoolbased intervention targeting marijuana use [27], the Teen Marijuana Check-Up (TMCU), since empirically validated [28-30] and designated as an evidence-based practice [31]. Table 1 lists its core characteristics.

\section{A key challenge for implementing TMCU in schools}

As in other health fields, experimental controls inherent in adolescent treatment trials limit generalizability of resulting findings [32]. This is prominent among reasons for 'voltage drop', where effect size diminishes upon a therapy's community implementation [33]. Though public demand for school-based services like TMCU is strong, the prospect of real-world TMCU implementation raises concerns about identification, training, and oversight of school-based personnel. Extant research on MI training conducted with a diverse workforce documents: (1)

Table 1 Intervention characteristics of the teen marijuana check-Up

\begin{tabular}{ll}
\hline Intervention characteristic & \\
\hline Therapeutic foundation & Motivational interviewing \\
Target population & Teens, aged 14-19 years \\
Nature of recruitment & Self-referral \\
Implementation setting & High schools \\
Intervention format & Brief individual therapy \\
Intervention duration & Two 60-min sessions, scheduled \\
& approximately 1 week apart \\
Core features & Patient-centered communication, \\
& review of a personalized feedback \\
report \\
Primary outcomes & Decreased marijuana use, reduced \\
marijuana-related harms \\
Secondary outcomes
\end{tabular}

TMCU characteristics as developed in or informed by a series of prior efficacy trials [31-34] 
insufficiency of workshop training to cull durable skills $[34,35]$, and (2) utility of post-workshop technical assistance via coaching and performance-based feedback [36-38]. While such technical assistance is broadly recommended to disseminate health services [39-41], key questions for schools at which TMCU is implemented are how much technical assistance personnel require.

Given prominent concern about costs [42, 43], much may be gained from identifying affordable strategies to avail technical assistance [44]. In efficacy trials, goldstandard technical assistance includes fidelity monitoring, provision of feedback on work-samples, and recurrent behavioral rehearsal opportunities [45] -all to assure research therapists maintain sufficient fidelity [46]. Such gold-standard technical assistance was included for research therapists in TMCU efficacy trials [27-30]. Given scant resources at many schools, the prospect of replicating such procedures in support of TMCU implementation is understandably daunting. A consequent challenge is to identify alternative strategies whereby technical assistance is offered to resource-challenged schools without compromise to TMCU fidelity [47].

\section{Process control benchmarking: a promising TMCU implementation support strategy}

One potential option for affordable, effective technical assistance in implementing the TMCU is governance by process control benchmarking. This involves continual tracking of interventionist fidelity and comparison to a priori benchmarks [48], whether conceptually derived performance standards [49], norms derived from a clinical database [50], or aggregated efficacy trial data [51]. For the TMCU, this comparative process will be facilitated by existence of conceptually derived performance standards for MI/MET interventions [52] and fidelity data from efficacy trials encompassing a dozen research therapists and nearly 700 students [27-30].

A testable implementation support strategy utilizing process control benchmarking, aforementioned MI/MET performance standards, and prior TMCU efficacy trial data is a fidelity drift alarm [48], illustrated in Fig. 1. As school-based interventionists are subject to natural performance variance due to setting demands, student casemix, and situational factors, a TMCU fidelity drift alarm would signal points of deviation from acceptable fidelity parameters, with an alarm bell prompting individually focused technical assistance. If occurring situationally, or 'as-needed', this is likely to diminish required resources. A fidelity drift alarm may be maintained (and re-calibrated, if appropriate) over time, thus allowing malleable and continual fidelity-monitoring. Relative to gold-standard technical assistance, this may be viable as a clinical and cost-effective method of technical assistance for schools to transition to lesser purveyor reliance. Moreover, it represents a sustainment strategy that may be applied to other empirically supported therapies implemented in schools.

The trial design described herein reflects a protocol funded by the National Institute on Drug Abuse (R01 DA040650, A Hybrid Effectiveness-Implementation Trial of a School-Based Teen Marijuana Check-Up). A hybrid type II trial [53], co-primary aims are to compare technical assistance methods for school-based TMCU interventionists on implementation outcomes and intervention effectiveness. School leaders will identify staff to be trained and serve as interventionists, then randomized to receive technical assistance over a 2-year period via (1) a 'goldstandard' group session occurring weekly, or (2) 'asneeded' individual sessions, triggered by a fidelity drift alarm calibrated by process control benchmarking. Selfreferring students will be randomized, in 2:1 ratio, to TMCU or waitlist/control. Hypothesized effects are for

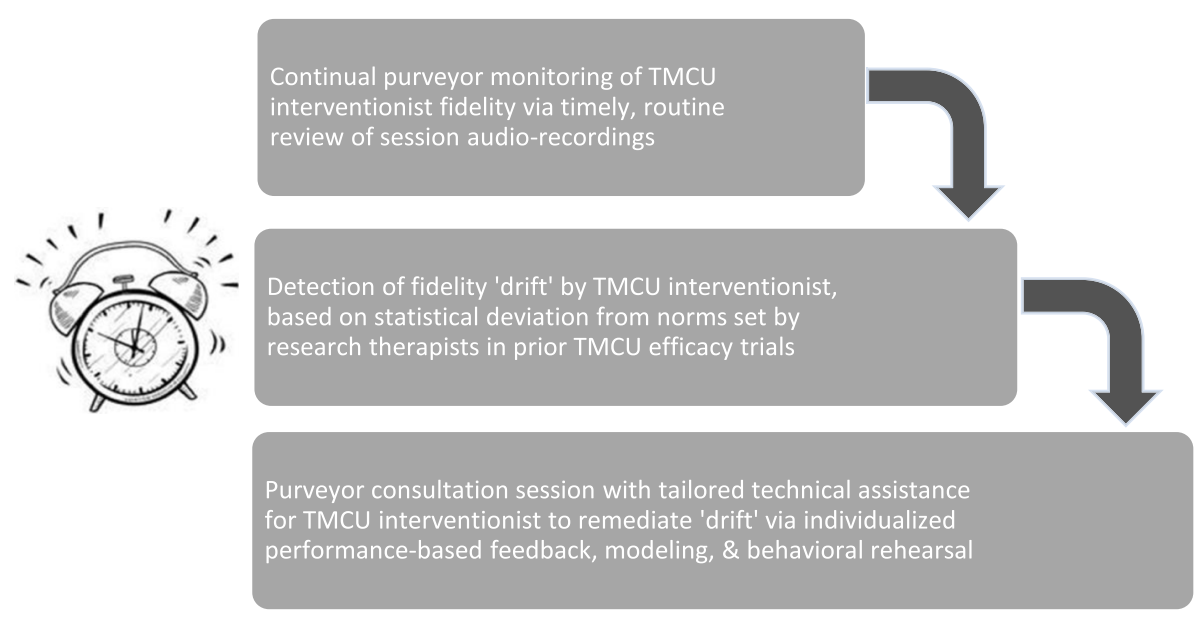

Fig. 1 Fidelity drift alarm 
non-differential impact of technical assistance methods on interventionists' TMCU fidelity and marijuana use among TMCU-exposed students. Other expectations are for lesser cost of 'as-needed' technical assistance, and clinical effectiveness of TMCU relative to waitlist/ control.

\section{Methods/design}

This hybrid type II effectiveness/implementation trial models the 'Exploration, Preparation, Implementation, Sustainment' (EPIS) framework [54]. This includes: mixed-method exploration of school contexts, preparation of school-based staff via formally evaluated TMCU training, post-training implementation over 24 months wherein trained TMCU interventionists are randomized to receive one of two technical assistance methods, and examination of sustainment via interventionist completion of biannual outcome assessments. As legislative and sociocultural factors converge in Washington state that heighten adolescent exposure to potential marijuanarelated harms, this trial seeks to advance knowledge of best implementation practices for this and other schoolbased interventions.

\section{Units of analysis and measures}

Trial measurement includes students nested in interventionist caseloads, which in turn are nested in schools. Units of analysis exist at three levels: school, interventionist, and student. Corresponding data collection instruments are listed in Table 2.

\section{Schools}

Trial measurement at a school-level is governed by a mixed-method approach, predominantly initially assessment of school attributes. For an estimated ten participating high schools, this includes web-based surveys of

Table 2 Trial measurement instruments and processes

\begin{tabular}{|c|c|c|c|}
\hline Unit of analysis & Instrument/process & Respondent(s) & Timing of assessment \\
\hline \multicolumn{4}{|l|}{ Schools } \\
\hline & Implementation leadership scale [62] & School staff members & Trial outset \\
\hline & Implementation climate scale [63] & School staff members & Trial outset \\
\hline & Site visit including elicitation interview with school leadership & Principal, other leaders & Trial outset \\
\hline & Exit interview & Principal, other Leaders & Trial conclusion \\
\hline \multicolumn{4}{|l|}{ Interventionists } \\
\hline & Demography and background & Interventionists & Pre-training \\
\hline & MI knowledge/attitudes test [64] & Interventionists & Pre/post-training +6-, 12-, 18-, 24-month \\
\hline & Adoption readiness scale [65] & Interventionists & Pre/post-training +6-, 12-, 18-, 24-month \\
\hline & Fidelity-rated interaction with a standardized patient [66] & Interventionists & Pre/post-training +6-, 12-, 18-, 24-month \\
\hline & Training satisfaction survey & Interventionists & Post-training \\
\hline & TA satisfaction survey & Interventionists & $6-, 12-$, 18-, 24-month \\
\hline & Interventionist time-log & Interventionists & Weekly, over 24 months \\
\hline \multicolumn{4}{|l|}{ Students } \\
\hline & Demography and locator data & Students & Baseline \\
\hline & Normative perceptions $[67,68]$ & Students & Baseline \\
\hline & Life goals [69] & Students & Baseline \\
\hline & Global Appraisal of Independent Needs [70] & Students & Baseline, 3- and 6-month \\
\hline & Marijuana Problems Scale [71] & Students & Baseline, 3- and 6-month \\
\hline & Marijuana Motives Measure [72] & Students & Baseline, 3- and 6-month \\
\hline & Self-efficacy to Avoid Marijuana [73] & Students & Baseline, 3- and 6-month \\
\hline & Student Academic Self-Report [74] & Students & Baseline, 3- and 6-month \\
\hline & Student Engagement Instrument [75] & Students & Baseline, 3- and 6-month \\
\hline \multicolumn{4}{|l|}{ Investigators } \\
\hline & Technical Assistance Time-Log & Purveyor & Weekly, over 24 months \\
\hline & Stages of Implementation Completion Checklist [76] & Research coordinator & Continual \\
\hline
\end{tabular}

Trial design proposes recruitment of approximately ten schools, with 2-4 interventionists recruited per participating school to garner an intended sample of TMCU interventionists $(N=30)$ randomized to 'gold-standard' or 'as-needed' technical assistance conditions; Self-referring students $(N=250)$ will be randomized in a 2:1 ratio to receive the TMCU intervention vs. waitlist/control 
strategic leadership [55] and implementation climate [56] completed by school staff (not restricted to those serving as TMCU interventionists). This is supplemented by school data necessary for determination of TMCU costs (i.e., existing resources for substance use programming; interventionists' salary; required facilities, equipment, supplies). This data will be gathered qualitatively in an initial elicitation interview with school leadership. Similar interviews are conducted at trial conclusion with school leadership and other personnel, focused on perceived facilitators and barriers for TMCU sustainment.

\section{Interventionists}

Trial measurement for $30 \mathrm{TMCU}$ interventionists to be recruited ( $n=2-4$ per school) includes pre-training collection of demography (age, gender, ethnicity, race), professional background (educational attainment; setting tenure, role, duties), and prior MI exposure. Repeatedmeasures assessed in six training outcome assessments (i.e., pre/post-training; 6-, 12-, 18-, 24-month follow-up) are the survey-based Motivational Interviewing Knowledge and Attitudes Test [57] and Adoption Readiness Scale [58], which supplement an audio-recorded standardized patient (SP) interaction conducted in interventionists' workspace and independently scored with a validated fidelity scale [59] by raters blinded to technical assistance condition. Post-training assessment specifically adds a web-based survey of training satisfaction, with follow-up assessments similarly tapping satisfaction with technical assistance and potential contamination between technical assistance methods. To aid planned cost analyses, interventionists will submit weekly time-logs to document effort in core implementation activities (i.e., hours spent recruiting students and delivering the intervention). Interventionists will receive financial remuneration to incentivize retention and completion of assessments.

\section{Students}

Trial measurement of 250 high school students to self-refer for randomization (in 2:1 ratio) to TMCU or waitlist/control includes baseline survey of demography, contact information, normative perceptions of marijuana use [60,61], and life goals information [62] incorporated as personal feedback. Repeated-measures assessed in intervention outcome assessments (i.e., baseline, 3- and 6-month follow-up) include the following student-report instruments (1) Global Appraisal of Independent Needs [63], offering recent marijuana use and diagnostic symptoms; (2) Marijuana Problems Scale [64]; (3) Marijuana Motives Measure [65]; (4) self-efficacy to avoid marijuana use [66]; (5) Student Academic SelfReport, tapping homework completion, attendance, and disciplinary actions [67]; and (6) Student Engagement
Instrument [68], measuring academic and social engagement. This will be supplemented by administrative data accessed from participating school districts. Students will receive financial remuneration to incentivize retention and completion of outcome assessments.

\section{Other measurement}

Trial measurement includes two staff-report instruments. To facilitate cost analyses, the TMCU purveyor (DW) will over the 24-month implementation period complete a weekly time-log of technical assistance efforts. Also, a Stages of Implementation Completion checklist [69], will be continually updated by the trial coordinator (LM) with record of milestone dates at participating schools for engagement, feasibility determination, trial planning, interventionist training, introduction of technical assistance, initiation of TMCU services, fidelity-monitoring, school decisions about sustainment.

\section{Trial procedures}

A procedural chronology begins with initial exploration of participating school contexts. This involves recruitment of schools, assessment of their setting attributes, and identification of staff candidates to serve as TMCU interventionists.

\section{School recruitment}

An initial step to access the intended sample of 30 interventionists is recruitment of schools, a process governed by the TMCU purveyor (DW) with whom local schools previously collaborated. Initially, school district-level approval will be sought, followed by contacts to school principals. Although no formal inclusion/exclusion criteria constrain this process, common contextual conditions are expected (i.e., need for marijuana-focused student services, interested and capable staff to serve as interventionists). The number of schools recruited is guided by access to a sufficient number of interventionists $(N=30)$ for planned comparison of 'gold-standard' and 'as-needed' technical assistance. Consideration is also given to schools' census, so a requisite number of self-referring students $(N=250)$ is achieved.

\section{Assessment of school attributes}

Two investigative team members (AL, DW) will schedule a site visit to observe setting attributes germane to TMCU implementation costs and conduct an audio-recorded elicitation interview with school leadership. The purpose of the interview is to (1) confirm understanding of trial aims and procedures; (2) gather information concerning school mission and resources, organizational climate and culture, structure and size of existing staff, and expectations and concerns about TMCU implementation; and (3) elicit a shortlist of TMCU interventionist candidates and 
their salary information. School leaders will review and sign a consent form prior to the interview. Interview recordings will be coded by the investigative team using conventional and directed content analysis [70]. Interested school staff will review and sign a consent form and complete a web-based survey tapping perceptions of strategic leadership [55] and implementation climate [56].

\section{Identification of TMCU interventionists}

Identified TMCU interventionist candidates will attend an on-site presentation. In addition to orientation to TMCU, the presentation will outline trial benefits (i.e., purveyor-led training and technical assistance, continuing education units) and expectations (i.e., completion of serial training outcome assessments, school designation as a TMCU interventionist, on-site promotion, screening and intervention, session audio-recording, submission of time-logs). Interested staff will be invited to become TMCU interventionists and asked to review and sign a corresponding consent form. Figure 2 offers a CONSORT flow diagram depicting trial procedures for TMCU interventionists.

The second EPIS phase involves preparation for schoolbased staff to serve as TMCU interventionists, for whom broad inclusion criteria should increase generalizability of trial findings. This includes their completion of a pretraining assessment, workshop training plus training cases, and a post-training assessment. Trained staff will then be randomized to one of two technical assistance methods.

\section{Pre-training assessment}

Interventionists will complete a web-based survey assessment of personal demography and professional background, MI knowledge and attitudes, and adoption readiness. Interventionists will also complete an audiorecorded encounter with a standardized patient (SP), who remains in character during portrayal of a marijuanausing teen. As in all training outcome assessments, the trial coordinator (LM) will coordinate scheduled times for SP encounters, with resulting audio-recordings independently scored for fidelity.

\section{Workshop training}

Identified staff will complete workshop training with a curriculum to mirror that used in TMCU efficacy trials. Readings will be provided in advance, to include research on marijuana and its effects, the TMCU manual, and an MI text [71]. Two investigators (BH, DW), both longstanding Motivational Interviewing Network of Trainers (MINT [72]) members, will facilitate a 2-day workshop with didactic review of MI principles and the TMCU manual, modeling of techniques, and behavioral rehearsal exercises. The initial workshop day is devoted to research findings on marijuana, risk factors for substance abuse, and introduction to MI principles. The latter workshop day focuses on the TMCU approach, with detailed review of the TMCU personal feedback report. Relevant MI skills will be introduced and practiced, with sample case vignettes offering further opportunities for TMCU interventionists to practice skills.

\section{Standardized training cases}

Following the workshop, each TMCU interventionist will complete training cases involving SP portrayal of a marijuana-using teen. Each case will be structured as a 30-45 min interaction, to occur at arranged times in interventionists' regular workspace. The workshop trainers (BH, DW) will score corresponding audio-recordings with an MI fidelity instrument [73], providing written feedback on performances relative to these conceptually derived behavioral fidelity benchmarks (1) reflection-to-question ratio (R:Q), (2) percent open questions (\%OQ), and (3) percent complex reflections (\%CR).

\section{Post-training assessment}

Upon training conclusion, interventionists complete a web-based survey assessment of MI knowledge and attitudes, adoption readiness, and training satisfaction. Additionally, interventionists will repeat the audio-recorded SP encounter to assess the impact of training on TMCU fidelity.

\section{Staff randomization}

At training conclusion, the trial coordinator (LM) will allocate via urn randomization TMCU interventionists to receive technical assistance via (1) a 'gold-standard' weekly group session, or (2) 'as-needed' individual sessions triggered by fidelity drift alarm. A shared feature of both methods is the direct oversight of the TMCU purveyor (DW), who will monitor fidelity in review of session audio-recordings and provide numeric feedback to interventionists regarding behavioral fidelity indices (i.e., R:Q, $\% O Q, \% C R)$. Notably, the technical assistance methods differ in format and anticipated frequency of occurrence, with differential cost expected.

The third EPIS phase focuses on TMCU implementation at participating schools over a 24-month period. This includes recruitment and randomization of selfreferring students, baseline student assessment, TMCU delivery, fidelity-monitoring and technical assistance, and student outcome assessments (3-, 6-month followups). To inform cost-benefit and cost-effectiveness analyses, both purveyor and interventionists complete weekly continual time-logs documenting effort in select activities. Figure 3 offers a CONSORT flow diagram depicting trial procedures for self-referring students. 


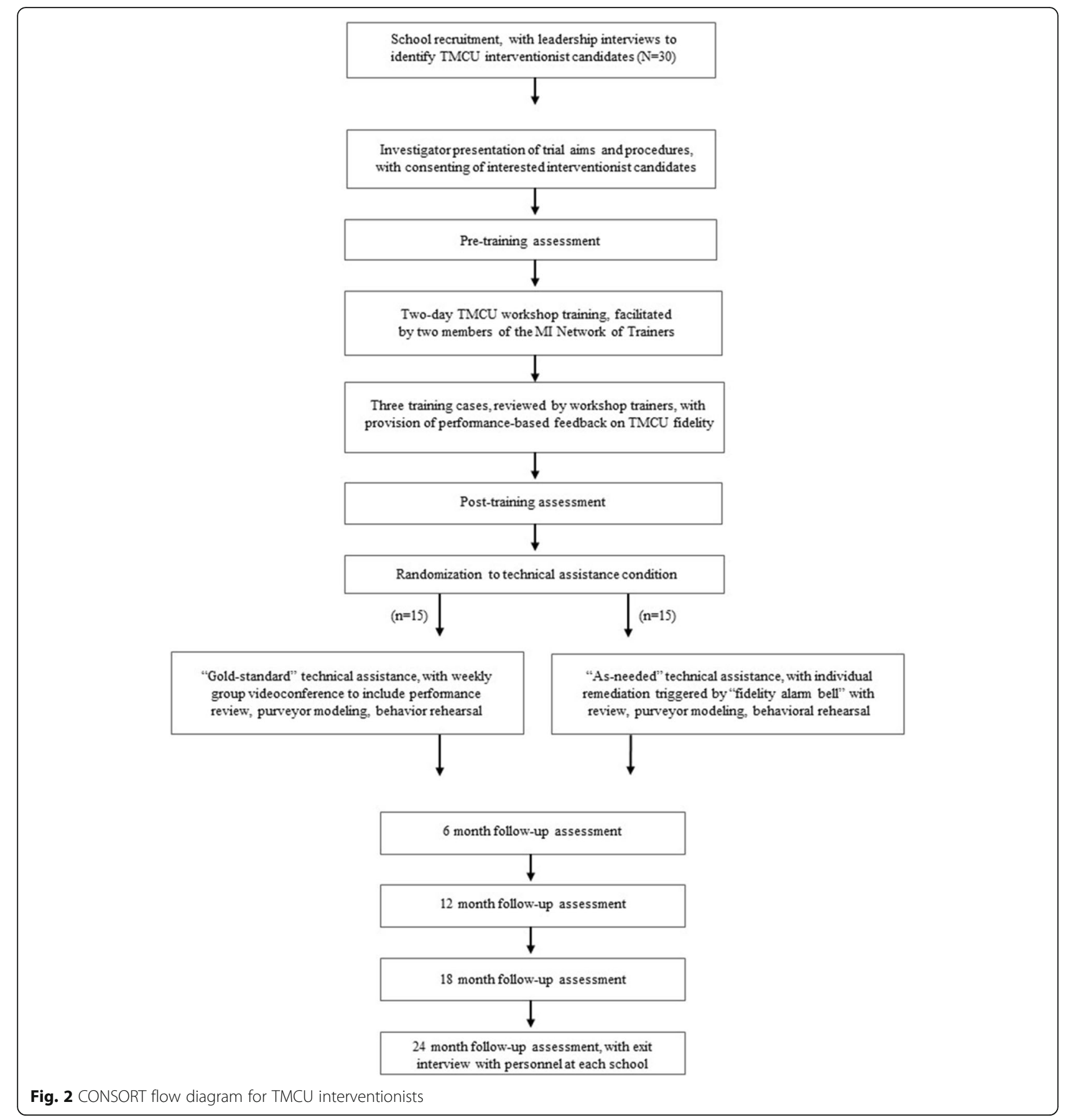

\section{Student recruitment}

At each school, TMCU interventionists will contribute to on-site promotion via classroom presentations and cafeteria exhibits. Self-referring students will have a TMCU interventionist read a brief explanation of the trial, and if interested will be directed to a web-based assent/consent form with contact information for university-based research staff. Students will provide informed assent/consent prior to interventionist conduct of a brief, confidential screening interview to determine eligibility. Inclusion criteria are that students be (1) 14-19 years old, (2) enrolled in 9th-12th grade, and (3) active marijuana users ( $9+$ days in prior month). Students will be excluded if they are not fluent in English, have a thought disorder, or refuse to be randomized to condition. Ineligible students will receive standard marijuana resources/referrals.

\section{Student randomization}

Upon determination of eligibility, students will be randomized in a 2:1 ratio to TMCU or waitlist/control. This 


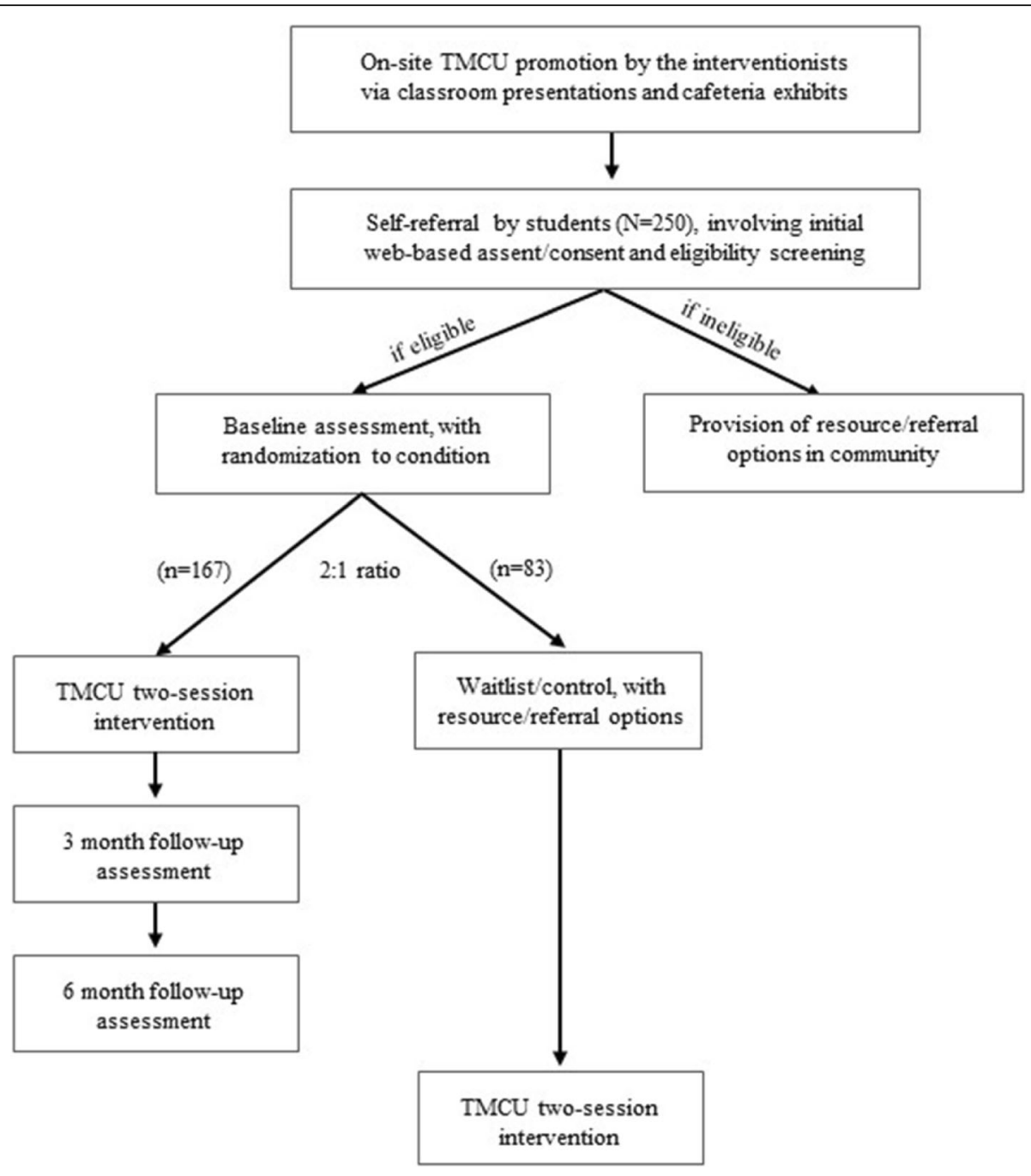

Fig. 3 CONSORT flow diagram for students

will occur after blocking by student gender and gradelevel, independently at each school. The trial seeks 250 student participants during its 24-month period of TMCU implementation.

\section{Baseline student assessment}

All student assessments occur over encrypted web servers. Baseline assessment will gather demography, contact information, and TMCU personal feedback report data on marijuana use, problems, motives, perceptions, and selfefficacy to abstain.

\section{TMCU delivery}

Trained interventionists will deliver the TMCU to eligible, self-referring students over a 24-month period. This consists of two sessions, each $45 \mathrm{~min}$ in duration occurring a week apart. The initial session develops rapport, explores aspects of the student's marijuana use, and identifies sources of motivation to change. The latter session includes conjoint review of the personal feedback report, with the interventionist eliciting self-motivational statements about reduction or discontinuance of marijuana use and offering advice for such changes. Notably, interventionists deliver TMCU to waitlist-assigned students only after a requisite 6-month intervening period.

\section{Purveyor fidelity-monitoring}

All TMCU sessions will be subject to fidelity-monitoring by the purveyor $(\mathrm{DW})$. Fidelity will be evaluated via a behavioral coding system with demonstrated validity for evaluating adherence of MI-based interventions [73]. It includes continuous counts of specific interventionist behaviors from which three focal behavioral fidelity indices are computed (i.e., R:Q, \%OQ, \%CR). 


\section{'Gold-standard' technical assistance}

Modeled after efficacy trial procedures, TMCU interventionists assigned to this condition will attend a weekly videoconference. The purveyor (DW) will lead discussion of her review of behavioral fidelity indices and excerpts of recently conducted sessions. If interventionist drift is identified in a reviewed session, this will prompt (1) conceptual review of relevant TMCU skills, (2) purveyor demonstration, and (3) behavioral rehearsal by the interventionist.

\section{'As-needed' technical assistance}

The purveyor (DW) will initiate individual sessions by videoconference at points when the fidelity alarm bell is triggered by unacceptable drift in one or more behavioral fidelity indices (i.e., R:Q, \%OQ, \%CR). The alarm will be calibrated using process control benchmarking, with interventionist performances compared against index distributions of research therapists from efficacy trials. For each fidelity index, these distributions will specify an a priori lower control limit to reflect the poorest acceptable TMCU fidelity. Interventionist performances that breach such limits will trigger the alarm, prompting 'as-needed' remediation. Technical assistance activities will mirror those of 'gold-standard' sessions (i.e., conceptual review, purveyor demonstration, interventionist behavioral rehearsal).

\section{Student outcome assessments}

The trial coordinator will govern procedures for these assessments at 3- and 6-month follow-up points, for which the purpose is to evaluate TMCU's clinical effectiveness. Students will similarly complete follow-up webbased assessments on encrypted internet server. Attrition will be minimized through use of extensive contact/locator information by research staff, who maintain networking relationships with local social service agencies. Similar procedures have led to $90+\%$ retention rates in prior TMCU trials.

\section{Purveyor/interventionists time-logs}

To inform eventual cost analyses, the purveyor and interventionists submit weekly time-logs during the 24-month implementation period. The purveyor documents effort in fidelity-monitoring, individual/group technical assistance, and crisis consultation or other interventionist support activities. The TMCU interventionists document effort recruiting students, delivering TMCU sessions, and attending technical assistance sessions.

The final EPIS phase addresses potential sustainment of TMCU at participating schools. This includes biannual outcome assessments completed by TMCU interventionists, cost-benefit and cost-effectiveness analyses, and elicitation interviews with school leadership.

\section{Training outcome assessments}

All interventionists complete biannual training outcome assessments (i.e., 6-, 12-, 18-, and 24-months after training). Like preceding assessments, each involves completion of an audio-recorded SP interaction to measure TMCU fidelity, and web-based survey assessment of TMCU knowledge, attitudes, and adoption readiness. A novel component of these follow-up assessments is the inclusion of interventionist-reported satisfaction with technical assistance they receive.

\section{Exit interviews}

At conclusion of 24-month implementation, two investigative team members (AL, DW) will conduct an elicitation interview with leaders and interventionists at each school. Setting facilitators and barriers to TMCU sustainment will be explored. Like initial interviews with school leadership, exit interviews will be audio-recorded and subject to conventional and directed content analysis [70].

\section{Cost analyses}

Examination of the value and relative affordability of the two technical assistance conditions will be governed by an investigative team member (KM) with expertise in economic evaluation. This involves use of a standardized survey [74] to estimate annual TMCU costs, incorporating student case-flow data (i.e., average daily student census, number of TMCU sessions delivered) to determine annual cost per student. In comparing costs of the technical assistance methods, data will correspond to aggregate resource use associated with TMCU implementation-including incremental costs associated with each method. Cost analyses will be framed from the school perspective, thus research costs will be excluded. Final products of the analysis will be estimates of total TMCU cost, respective incremental costs of each technical assistance method, annual cost per student, and average cost per TMCU intervention (based on mean number of TMCU sessions completed).

Cost estimates will then be linked with key outcomes to determine cost-benefit and cost-effectiveness. Costeffectiveness analysis compares differences in the incremental cost of TMCU to differences in its incremental effectiveness in reducing marijuana use to calculate the incremental cost-effectiveness ratio. This ratio describes the additional cost to achieve a unit of desired outcome (i.e., cost per one fewer day of student marijuana use) in TMCU relative to waitlist/control. Cost-benefit analysis broadens the economic impact assessment by comparing intervention costs to the monetary value of reducing negative consequences (i.e., school absences, criminal activity, medical costs, and lost productivity among parents/caregivers. Monetary conversion factors for units of outcome 
(e.g., cost per criminal offense or cost per day missed from work) translate such outcomes into dollars, which can then be directly compared to intervention costs. The costbenefit ratio expresses the dollars of benefit generated per dollar invested in the intervention.

\section{Data management}

Trial data consist of audio-recordings and web-based surveys. Audio-recordings include elicitation interviews of school leadership, SP interactions completed by TMCU interventionists, and TMCU sessions with participating students. Web-based surveys are completed by TMCU interventionists, other school-based staff, and students. Upon their collection, audio-recordings will be transported immediately to secure university office space and uploaded as audio-files to a password-protected network and identified by appropriate trial identification number. Original recordings will then be deleted from digital recording devices. Upon their completion, web-based surveys will be stored on encrypted university servers accessible only by trial staff, similarly identified by trial identification number. As outlined in a corresponding Data Safety and Monitoring Plan, linkage between identifying information for trial participants and trial identification numbers will be destroyed at trial conclusion.

Planned reliance on web-based surveys for most of the trial data collection limits the need for data entry procedures. An exception is paper forms corresponding to audio-recording data (i.e., TMCU fidelity ratings), for which numeric data will be entered and later reviewed for verification by separate study staff. Likewise, automated entry of web-based survey data will be reviewed for verification by study staff who will identify and fix anomalies. Record of data anomalies will be maintained in a trial logbook. To facilitate data completeness, a data tree will be established in order that data is analyzed and stored in specific files, backed up in secure locations on a weekly basis.

\section{Hypothesis testing and analytic approach}

Hypotheses focus on comparative utility of two technical assistance methods on implementation outcomes among TMCU interventionists and intervention effectiveness among students. Hypothesized effects are that the (1) longitudinal effects of interventionists receiving 'goldstandard' vs. 'as-needed' technical assistance will not differ, with the latter method proving more cost-beneficial and cost-effective; and (2) effects of technical assistance method for intervention impact on student marijuana use frequency will not differ, with greater reduction expected of students randomly assigned to TMCU vs. waitlist/control.

Trial analyses will be governed by an investigative team member (KK), experienced as a biostatistician and methodologist in adolescent health research. Given nesting of trial data (i.e., repeated observations of students, nested in interventionist caseloads; interventionists nested in schools), multilevel models will include progressive taxonomies in model-building. Initially, an unconditional model will estimate variance of random effects, and a subsequent unconditional growth model will identify the appropriate shape of and variability in rate of change over time for hypotheses involving repeated measures. Technical assistance condition will then be included in conditional models with predictors, with relevant covariates added to determine robustness of main effects and relative goodness-of-fit for each successive model via likelihood ratio tests and deviance statistics. Power analyses $(.80, p<.05)$ inform proposed recruitment of (1) 30 interventionists to detect longitudinal effects of training and technical assistance consistent with those for other lay counselors (Cohen's $d=1.00$ ); and (2) 250 students to detect small intervention effects (Cohen's $d=.35$ ) relative to waitlist/control.

\section{Hypothesis 1a: comparable utility of technical assistance methods}

A non-inferiority paradigm, traditionally used to show a novel treatment is not statistically different than an established one, will test study hypotheses. This paradigm will be applied to interventionists' longitudinal implementation outcomes, principally TMCU fidelity (i.e., $\mathrm{R}: \mathrm{Q}, \% \mathrm{OQ}, \% \mathrm{CR}$ ) in SP interactions but also secondary outcomes of TMCU knowledge, attitudes, adoption readiness, and satisfaction. Initial effects are consistent with training intent, with analyses testing if 'gold-standard' and 'as-needed' technical assistance produce equivalent longitudinal impacts. If (as expected) the number of schools recruited is small, they will be dummy-coded and treated as fixed effects. Point-estimates and confidence intervals from the model will be included in non-inferiority tests, examining each technical assistance method for (1) prepost change in implementation outcomes, (2) mean implementation level by TMCU interventionists over time, and (3) differential rate of change in implementation over time. As recommended [75], a 1/2 standard deviation (SD) difference will serve as the a priori noninferiority margin. If the lower bound of two-sided 95\% confidence interval of between-method differences exceeds this margin, a test of superiority will specify the direction and magnitude of differential impact of the technical assistance methods.

\section{Hypothesis 1b: comparable clinical effectiveness of technical assistance methods}

For student outcomes, multilevel models extend to three levels for repeated observation of marijuana use frequency and secondary outcomes (i.e., problems, motives, 
perceptions, self-efficacy to abstain, academic performance, and engagement) nested within interventionists. A primary test parameter is the interaction of time $\times$ technical assistance method of the TMCU interventionist as a dummy-coded predictor. As temporal effects may not conform to simple linear growth curves, models will test different specifications of time (i.e., piecewise, polynomial, or other nonlinear approaches). The a priori noninferiority margin (i.e., $1 / 2 \mathrm{SD}$ ) will again be applied and, if exceeded, a test of superiority will specify the direction and magnitude of differential impact.

\section{Trial regulation and current status}

In accordance with National Institute on Health policy, a Data Safety and Monitoring Board (DSMB) was assembled to whom the principal investigators $(\mathrm{BH}$, DW) recurrently report trial progress. Content expertise of this three-member, university-based DSMB encompasses school-based health service implementation, brief interventions, and community-participatory addiction research. The trial coordinator (LM) received approval for all procedures from the University of Washington Institutional Review Board to whom protocol modifications and adverse events will be reported, obtained a Certificate of Confidentiality, and registered the trial at ClinicalTrials.gov (NCT03111667). As for trial progress, recruitment of local schools has been initiated with formal enrollment of TMCU interventionists expected in the months to come.

\section{Discussion}

As schools are a common point-of-contact for students in the USA to access behavioral health services [21, 22], there are several reasons this has potential to address marijuana use. First, most adolescents who misuse substances evidence marijuana use disorder [7], a leading impetus for youth treatment admissions [8]. Second, progressive legislation of medical/recreational marijuana use widens its public availability and cultural permissibility [13], while risk perceptions among high school students continually decline [12]. Third, over $95 \%$ of teens with a substance use disorder continue to attend school-so school-based services should offer significant reach [76]. Taken together, these issues underscore need for effective, affordable strategies to support transport of efficacious, marijuana-focused health services for use by school-based personnel. The described trial-and its testing of a fidelity alarm bell as a method of purveyor technical assistance-is designed to offer one such effort. Resulting trial findings are likely to hold implications for similar service transportability challenges across a range of health services and the community-based settings for which they are appropriate.

\section{Innovation and potential impact}

The described trial offers innovation at multiple levels. Most broadly, its findings will address a salient gap in implementation science literature through examination of processes for purveyor introduction and support of an empirically supported health service. Though this trial will specifically examine implementation of a brief marijuana-focused intervention in schools, replication of many design features described herein may be appropriate for other trials. At the level of implementation strategies, this trial will test a novel approach whereby technical assistance is provided to school-based staff after initial training. As technical assistance processes remains an understudied topic [77], a clinically useful, affordable means to provide such support may hold considerable impact for therapy purveyors and community settings. Notably, the 'fidelity alarm bell' to be tested retains core elements (i.e., purveyor fidelity monitoring, feedback, coaching) recommended for implementing MI/MET [41] and other therapies [39, 40]. A final point concerns potential future interplay with ongoing validation work on automated MI/MET fidelity assessment methods that code session recordings via computer and transform fidelity indices as visual feedback [78]. If feasible, future work may pair a fidelity alarm bell with these promising natural language processing approaches to streamline fidelity-monitoring processes [79-81] and thereby boost the efficiency and scale with which they occur. Diminished purveyor burden in this area may open possibilities to enhance feedback/coaching processes [82], or simply reduce purveyor reliance in favor of localized, self-sustaining quality assurance methods [48].

\section{Limitations}

Design caveats bear mention. Potential for selection bias exists for participating schools, interventionists, and students. Recruitment of schools that collaborated on prior TMCU trials may undermine generalizability of results, though both the multisite design and broad inclusion criteria for settings and interventionists mitigate this concern. With respect to TMCU effectiveness, student inclusion/exclusion criteria are consistent with those of prior efficacy trials and the intent of this targeted intervention [27]. The trial responds to local demands in Washington state, where legalization of recreational marijuana use among adults has amplified public concern and demand for preventative services. Persisting variation in legislative marijuana policy, both domestically and abroad [83], suggests this local context be considered in eventual interpretation of trial findings. Measurement choices present additional caveats. Trial aims necessitate audio-recording of TMCU sessions, which for some may introduce social anxiety and impression management. These concerns 
appear not to extend to planned SP interactions, given strong evidence for valid, reliable MI fidelity estimates via this methodology [84]. Reliance on student-report instruments to examine TMCU effectiveness is another caveat, particularly for marijuana use-though use of invasive biological testing with minors would introduce greater scientific and pragmatic concerns. As in any trial, potential influence of unassessed third variables is a possible limitation. Further, alternative trial designs (i.e., clusterrandomization, stepped-wedge) may offer some potential benefits in efficiency, albeit not without introducing other unwanted caveats.

\section{Conclusions}

This trial offers potential advancement for the implementation science field, specifically in its thorough examination of a data-driven technical assistance method to support long-term implementation of a marijuana-focused MET intervention in schools. If shown to be clinically useful and affordable, the concept of a fidelity drift alarm could be readily translated-alone or in future conjunction with emerging methods to automate MI/MET fidelity-monitoring-for use with other empirically supported therapies and in other settings. The described trial adds to other recent efforts [85] in furthering momentum for the adoption and effective implementation of efficacious therapies for youth populations through a focus on methods of purveyor technical assistance to assure quality in therapy delivery.

\begin{abstract}
Abbreviations
\%CR: Percentage of reflections deemed to be 'complex'; \%OQ: Percentage of questions deemed to be 'open'; DSMB: Data Safety Monitoring Board; EPIS: Exploration, Preparation, Implementation, Sustainment; MET: Motivational enhancement therapy; MI: Motivational interviewing; MINT: Motivational Interviewing Network of Trainers; R:Q: Ratio of reflections to questions; SD: Standard deviation; SP: Standardized patient; TMCU: Teen Marijuana Check-Up
\end{abstract}

\section{Acknowledgements}

The authors thank Meg Brunner, MLIS for her assistance in creating manuscript figures.

\section{Funding}

The described trial is funded by the National Institute on Drug Abuse, R01DA040650 A Hybrid Effectiveness-Implementation Trial of a School-Based Teen Marijuana Check-Up), awarded to Walker, D. and Hartzler, B. (multiple PIs). Additionally, preparation of this trial design paper was also supported by the noted award.

Availability of data and materials

Not applicable

\section{Authors' contributions}

All of the listed authors contributed to the design of the funded trial. BH drafted the manuscript and integrated all co-author editing. AL assisted in manuscript conceptualization. All authors reviewed, provided text and/or editing, and approved the final version of the manuscript. LM is the trial coordinator, and has attained all IRB approvals for the trial. All authors read and approved the final manuscript.
Ethics approval and consent to participate

All trial procedures have been reviewed by a local university-based institutional review board. Approval is by the University of Washington Human Subjects Division, Committee J.

\section{Consent for publication}

Not applicable

\section{Competing interests}

The authors declare that they have no competing interests.

\section{Publisher's Note}

Springer Nature remains neutral with regard to jurisdictional claims in published maps and institutional affiliations.

\section{Author details}

${ }^{1}$ Alcohol and Drug Abuse Institute, University of Washington, 1107 NE 45th Street, Suite 120, Seattle, WA 98105, USA. ${ }^{2}$ Psychiatry and Behavioral Sciences, University of Washington, 6200 NE 74th Street, Suite 100, Seattle, WA 98105, USA. ${ }^{3}$ School of Social Work, University of Washington, 909 NE 43rd Street, Suite 304, Seattle, WA 98105, USA. ${ }^{4}$ Department of Psychology, University of Washington, 119A Guthrie Hall, Seattle, WA 98195, USA.

${ }^{5}$ Department of Public Health Sciences, University of Miami Miller School of Medicine, 1120 NW 14th Street, Suite 1019, Miami, FL 33136, USA.

Received: 6 July 2017 Accepted: 3 August 2017

Published online: 10 August 2017

\section{References}

1. Grant J, Green L, Mason B. Basic research and health: a reassessment of the scientific basis for the support of biomedical science. Research Evaluation. 2003;12:217-24.

2. Balas EA, Boren SA. Managing clinical knowledge for health care improvement, in Yearbook of medical informatics, J.H. Van Bemmel and A.T. McCRay, Editors. 2000, Schattauer Verlagsgesellschaft mbH: Stuttgart, Germany. p. 65-70.

3. Wratchko K. The pharmaceutical industry: strategic orientation and alliance portfolio configuration 2009. New York: Springer.

4. Kendall PC, Beidas RS. Smoothing the trail for dissemination of evidence-based practices for youth: flexibility within fidelity. Prof Psychol Res Pract. 2007;38(1): $13-20$.

5. Croft B, Parish SL. Care integration in the patient protection and affordable care act: implications for behavioral health. Adm Policy Ment Health Ment Health Serv. 2013;40(4):258-63.

6. IOM, Bridging the gap between practice and research: forging partnerships with community-based drug and alcohol treatment. 1998: Washington.

7. Center for Behavioral Health Statistics and Quality, Behavioral health trends in the United States: Results from the 2014 National Survey on Drugs Use and Health, in NSDUH H-50. 2015. http://www.samhsa.gov/data. Accessed 20 June 2017

8. U.S. Department of Health and Human Services, Treatment Episode Data Set-Admissions (TEDS-A), 2010, Inter-university Consortium for Political and Social Research (ICPSR), Editor. 2014, Substance Abuse and Mental Health Services Administration, Center for Behavioral Health Statistics and Quality.

9. Meier $\mathrm{MH}$, et al. Persistent cannabis users show neuropsychological decline from childhood to midlife. Proc Natl Acad Sci U S A. 2012;109(40):E2657-64.

10. Silins $E$, et al. Young adult sequelae of adolescent cannabis use: an integrative analysis. Lancet Psychiatry. 2014;1 (4):286-93.

11. McGrath J, et al. Association between cannabis use and psychosis-related outcomes using sibling pair analysis in a cohort or young adults. Arch Gen Psychiatry. 2010;67(5):440-7.

12. Johnston $L D$, et al. Monitoring the future national results on drug use: 1975-2013: overview, key findings on adolescent drug use. 2014. Institute for Social Research, The University of Michigan: Ann Arbor, MI.

13. Pacula RL, et al. Developing public health regulations for marijuana: lessons from alcohol and tobacco. Am J Public Health. 2014;104(6):1021-8.

14. Project MATCH. Project MATCH (matching alcoholism treatment to client heterogeneity): rationale and methods for a multisite clinical trial matching patients to alcoholism treatment. Alcohol Clin Exp Res. 1993;17(6):1130-45. 
15. Anton RF, et al. Combined pharmacotherapies and behavioral interventions for alcohol dependence. THE COMBINE study: a randomized controlled trial. J Am Med Assoc. 2006;295(17):2003-17.

16. Hanson GR, Leshner Al, Tai B. Putting drug abuse research to use in real-life settings. J Subst Abus Treat. 2002;23:69-70.

17. Office of National Drug Control Policy. Substance abuse and the Affordable Care Act. 2014 [cited 2014 October 13].

18. Cabus SJ, De Witte K. Does school time matter? —on the impact of compulsory education age on school dropout. Econ Educ Rev. 2011;30(6): 1384-98.

19. Evans SW, Weist MD. Implementing empirically supported treatments in the schools: what are we asking? Clin Child Fam Psychol Rev. 2004;7(4):263-7.

20. Rones M, Hoagwood K. School-based mental health services: a research review. Clin Child Fam Psychol Rev. 2000;3(4):223-41.

21. Burns BJ, et al. Children's mental health service use across service sectors. Health Aff. 1995;14:147-59.

22. Farmer $E M Z$, et al. Pathways into and through mental health services for children and adolescents. Psychiatr Serv. 2003;54(1):60-6.

23. Miller WR, Rollnick S. Motivational interviewing: helping people change. 2013. New York: Guilford.

24. Miller WR. Motivational enhancement therapy with drug abusers. 1995. Albuquerque: University of New Mexico.

25. Miller WR, Sovereign RG, Krege B. Motivational interviewing with problem drinkers: the Drinker's check-up as a preventive intervention. Behav Psychother. 1988;16:251-68.

26. Walker DD, et al. The check-up: in-person, computerized, and telephone adaptations of motivational enhancement treatment to elicit voluntary participation by the contemplator. Substance abuse treatment, prevention, and policy. 2007;2:2

27. Berghuis JP, et al. The teen cannabis check-up: exploring strategies for reaching young cannabis users. In: Roffman RA, Stephens RS, editors. Cannabis dependence: its nature, consequences, and treatment. Cambridge: Cambridge University Press; 2006. p. 275-96.

28. Walker $\mathrm{D}$, et al. Motivational enhancement therapy for adolescent marijuana users: a preliminary randomized controlled trial. J Consult Clin Psychol. 2006; 74(3):628-32.

29. Walker DD, et al. Randomized controlled trial of motivational enhancement therapy with nontreatment-seeking adolescent cannabis users: a further test of the teen marijuana check-up. Psychol Addict Behav. 2011;25(3):474-84.

30. Walker DD, Stephens RS, Roffman RA. Preliminary results from the teen marijuana check-up: Testing the effect of motivational enhancement check-ins. Presented at the International conference on motivational interviewing; Amsterdam, Netherlands. 2014.

31. Lemon $\mathrm{M}$, et al. Preventing and treating youth marijuana use: an updated review of the evidence. 2014. Washington State Institute for Public Policy: Olympia.

32. Weisz JR, Doss AJ, Hawley KM. Youth psychotherapy outcome research: review and critique of the evidence base. Annu Rev Psychol. 2005;56:337-63.

33. Chambers $D$, Glasgow $R$, Stange $K$. The dynamic sustainability framework: addressing the paradox of sustainment amid ongoing change. Implement Sci. 2013:8(1).

34. Baer JS, et al. An evaluation of workshop training in motivational interviewing for addiction and mental health clinicians. Drug Alcohol Depend. 2004;73(1):99-106

35. Miller WR, Mount KA. A small study of training in motivational interviewing: does one workshop change clinician and client behavior? Behav Cogn Psychother. 2001;29:457-71.

36. Miller WR, et al. A randomized trial of methods to help clinicians learn motivational interviewing. J Counsel Clin Psychol. 2004;72(6):1050-62

37. Baer JS, et al. Context and tailored training in technology transfer: evaluating motivational interviewing training for community counselors. J Subst Abus Treat. 2009;37(2):191-202.

38. Moyers TB, et al. A randomised trial investigating training in motivational interviewing for behavioural health providers. Behav Cogn Psychother. 2008; 36:149-62.

39. Wiltsey Stirman S, et al. The sustainability of new programs and innovations: a review of the empirical literature and recommendation for future research. Implement Sci. 2012;7:17.

40. Tibbits MK, et al. Sustaining evidence-based interventions under real-world conditions: results from a large-scale diffusion progject. Prev Sci. 2010;11: 252-62.
41. Miller WR, et al. Disseminating evidence-based practices in substance abuse treatment: a review with suggestions. J Subst Abus Treat. 2006;31:25-39.

42. Herschell $A D$, et al. The role of therapist training in the implementation of psychosocial treatments: a review and critique with recommendations. Clin Psychol Rev. 2010;30:448-66.

43. Kazak $A E$, et al. A meta-systems approach to evidence-based practice for children and adolescents. Am Psychol. 2010;65(2):85-97.

44. McHugh RK, Barlow DH. The dissemination and implemenation of evidence-based psychological treatments: a review of current efforts. Am Psychol. 2010;65(2):73-84.

45. Baer JS, et al. Training and fidelity monitoring of behavioral interventions in multi-site addictions research. Drug Alcohol Depend. 2007;87(2007):107-18.

46. Proctor $\mathrm{E}$, et al. Outcomes for implementation research: conceptual distinctions, measurement challenges, and research agenda. Adm Policy Ment Health Ment Health Serv Res. 2011:38(2):65-76.

47. Garland AF, Bickman L, Chorpita BF. Change what? Identifying quality improvement targets by investigating usual mental health care. Adm Policy Ment Health Ment Health Serv. 2010;37:15-26.

48. Hogue A, et al. Making fidelity an intramural game: localizing quality assurance procedures to promote sustainability of evidence-based practices in usual care. Clin Psychol Sci Pract. 2013:20:60-77.

49. Weersing VR. Benchmarking the effectiveness of psychotherapy: program evaluation as a component of evidence-based practice. J Am Acad Child Adolesc Psychiatry. 2005;44:1058-62.

50. Mullin T, et al. Recovery and improvement benchmarks for counseling and the psychological therapies in routine primary care. Counsel Psychotherapy Res. 2006;6:68-80

51. Weersing VR, Weisz JR. Community clinic treatment of depressed youth: benchmarking usual care against CBT clinical trials. J Consult Clin Psychol. 2002:70:299-310.

52. Miller WR, et al. Manual for the Motivational Interviewing Skill Code (MISC) Version 2.1. 2008, University of New Mexico Center on Alcoholism Substance Abuse, and Addictions website: https://casaa.unm.edu/ codinginst.html. Accessed 20 June 2017.

53. Curran GM, et al. Effectiveness-implementation hybrid designs: combining elements of clinical effectiveness and implementation research to enhance public health impact. Med Care. 2012;50(3):217-26.

54. Aarons GA, Hurlburt M, McCue Horwitz S. Advancing a conceptual model of evidence-based practice implementation in public service sectors. Adm Policy Ment Health Ment Health Serv. 2011;38(1):4-23.

55. Aarons GA, Ehrhart MG, Farahnak LR. The implementation leadership scale (ILS): development of a brief measure of unit-level implementation leadership. Implement Sci. 2014;9(1):45.

56. Ehrhart MG, Aarons GA, Farahnak LR. Assessing the organizational context for EBP implementation: the development and validity testing of the implementation climate scale (ICS). Implement Sci. 2014;9(1):157.

57. Leffingwell TR. The motivational interviewing knowledge and attitudes test (MIKAT) for evaluation of training outcomes. Minuet. 2006;13:10-1.

58. McGovern MP, et al. A survey of clinical practices and readiness to adopt evidence-based practicies: dissemination research in an addiction treatment system. J Subst Abus Treat. 2004;26:305-12.

59. Moyers TB, et al. Assessing competence in the use of motivational interviewing. J Subst Abus Treat. 2005;28:19-26.

60. Neighbors C, Geisner IM, Lee CM. Perceived marijuana norms and social expectancies among entering college student marijuana users. Psychol Addict Behav. 2008;22(3):433-8.

61. Neighbors $C$, et al. Social identity as a moderator of the association between norms and marijuana use. J Stud Alcohol Drugs. 2013;74(3):479-83.

62. Emmons RA. Personal strivings: an approach to personality and subjective well-being. J Pers Soc Psychol. 1986;51(5):1058-68.

63. Dennis, M., Global appraisal of individual needs (GAIN): administration guide for the GAIN and related measures 1999. Bloomington: Chestnut Health Systems.

64. Stephens RS, Roffman RA, Curtin L. Comparison of extended versus brief treatments for marijuana use. J Consult Clin Psychol. 2000;68(5):898-908.

65. Simons J, et al. Validating a five-factor marijuana motives measure: Relations with use, problems, and alcohol motives. J Counsel Psychol. 1998;45(3):265-73.

66. Stephens RS, Wertz JS, Roffman RA. Self-efficacy and marijuana cessation: a construct validity analysis. J Consult Clin Psychol. 1995;63(6):1022-31.

67. Bruns $E B$, et al. Pilot test of an engagement, triage, and brief intervention strategy for school mental health. under review. 
68. Appleton JJ, et al. Measuring cognitive and psychological engagement: validation of the student engagement instrument. J Sch Psychol. 2006;44: $427-45$.

69. Chamberlain P, Brown CH, Saldana L. Observational measure of implementation progress in community based settings: the stages of implementation completion (SIC). Implement Sci. 2011;6:116.

70. Hsieh HF, Shannon SE. Three approaches to qualitative content analysis. Qual Health Res. 2005;15(9):1277-88.

71. Miller, W.R. and S. Rollnick, Motivational interviewing: helping people change (3rd edition). Applications of motivational interviewing. 2013, New York, NY, US: Guilford Press.

72. MINT. Motivational Interviewing Network of Trainers. 2017 [cited 2017 April 20]; Available from: http://www.motivationalinterviewing.org/.

73. Moyers TB, et al. Assessing competence in the use of motivational interviewing. J Subst Abus Treat. 2005;28(1):19-26.

74. French MT, et al. Outpatient marijuana treatment for adolescents: economic evaluation of a multisite field experiment. Eval Rev. 2003;27(4):421-59.

75. Wiens BL. Choosing an equivalence limit for non-inferiority or equivalence studies. Control Clin Trials. 2002;23:2-14.

76. Dennis ML, Clark HW, Huang LN. The need and opportunity to expand substance use disorder treatment in school-based settings. Adv School Ment Health Promot. 2014;7(2):75-87.

77. Kilminster SM, Jolly BC. Effective supervision in clinical practice settings: a literature review. Med Educ. 2000;34:827-40.

78. Atkins DC, et al. Scaling up the evaluation of psychotherapy: evaluating motivational interviewing fidelity via statistical text classification. Implement Sci. 2014;9(1):49.

79. Xiao B, et al. "Rate my therapist": automated detection of empathy in drug and alcohol counseling via speech and language processing. PLoS One. 2015;10(12):e0143055.

80. Can D, et al. "It sounds like......": a natural language processing approach to detecting counselor reflections in motivational interviewing. J Couns Psychol. 2016;63(3):343.

81. Tanana $\mathrm{M}$, et al. A comparison of natural language processing methods for automated coding of motivational interviewing. J Subst Abus Treat. 2016;65: 43-50.

82. Imel ZE, et al. Technology-enhanced human interaction in psychotherapy. $\mathrm{J}$ Couns Psychol. in press

83. Pacula RL, Sevigny EL. Marijuana liberalizations policies: why we can't learn much from policy still in motion. J Policy Anal Manag. 2014;33(1):212-21.

84. Imel ZE, et al. Evaluating therapist competence in motivational interviewing by comparing performance with standardized and real patients. J Consult Clin Psychol. 2014;82(3):472-81.

85. Dorsey $\mathrm{S}$, et al. Improving practice in community-based settings: a randomized trial of supervision - study protocol. Implement Sci. 2013;8:89.

\section{Submit your next manuscript to BioMed Central and we will help you at every step:}

- We accept pre-submission inquiries

- Our selector tool helps you to find the most relevant journal

- We provide round the clock customer support

- Convenient online submission

- Thorough peer review

- Inclusion in PubMed and all major indexing services

- Maximum visibility for your research

Submit your manuscript at www.biomedcentral.com/submit
Biomed Central 